\title{
Rationale and Methodology of The PopulatION HEalth and Eye Disease PRofile in Elderly Singaporeans Study [PIONEER]
}

\author{
Preeti Gupta $^{1}$, Ryan Eyn Kidd Man ${ }^{1}$, Eva K. Fenwick ${ }^{1,2}$, Amudha Aravindhan ${ }^{1}$, Alfred TL Gan ${ }^{1}$, \\ Sahil Thakur ${ }^{1}$, Bao Lin Pauline Soh ${ }^{3}$, Joanne M Wood ${ }^{4}$, Alex A Black ${ }^{4}$, Angelique Chan ${ }^{2}$, David \\ $\mathrm{Ng}^{2}$, Teoh Khim Hean ${ }^{5}$, Edwin Goh ${ }^{5}$, Chong Foong-Fong Mary ${ }^{6}$, Jenny Loo ${ }^{7}$, Ciaran Gerard \\ Forde $^{8}$, Charumathi Sabanayagam ${ }^{1,2}$, Ching-Yu Cheng ${ }^{1,2,9}$, Tien Yin Wong ${ }^{1,2,9}$, Ecosse L. \\ Lamoureux ${ }^{1,2,9 *}$
}

${ }^{1}$ Singapore Eye Research Institute and Singapore National Eye Centre, Singapore. ${ }^{2}$ Duke-NUS Medical School, Singapore. ${ }^{3}$ Singapore Institute of Technology, Health and Social Sciences, Singapore. ${ }^{4}$ Queensland University of Technology, Brisbane, Australia. ${ }^{5}$ National Dental Centre, Singapore. ${ }^{6}$ Saw Swee Hock School of Public Health, National University of Singapore, Singapore. ${ }^{7}$ National University Hospital, Singapore. ${ }^{8}$ Singapore Institute for Clinical Sciences, National University of Singapore, Singapore. ${ }^{9}$ Department of Ophthalmology, National University of Singapore, Singapore

[Received January 20, 2019; Revised February 3, 2020; Accepted February 6, 2020]

\begin{abstract}
To describe the rationale, design and methodology of a geographically-representative and population-based study investigating the epidemiology, impact, personal and economic burden of age-related eye diseases, declining visual and other sensory systems in Asians aged >60 years in Singapore.PIONEER (The PopulatION HEalth and Eye Disease PRofilE in Elderly Singaporeans Study) is currently a cross-sectional study targeting 3152 Chinese, Malay and Indian adults who are Singapore citizens or permanent residents aged 60 years and older living across Singapore. The study is intended to be longitudinal, with several waves of data planned to be collected in the future. The sampling frame consisted of $\mathbf{7 0 0 0}$ names derived from age, gender and ethnicity-stratified random sampling of individuals $>60$ years. Selected individuals were invited via letters, home visits, and telephone calls for a clinical assessment at the Singapore Eye Research Institute. Individuals with limited mobility were examined in a custom-designed mobile eye clinic. Questionnaires were subsequently administered at participants' homes by trained interviewers in their preferred language. A total of 3,299 participants (from East, West, North and South Singapore) were approached from December 2017 to November 2019. Of these, $953(28.5 \%)$ were deemed ineligible. Out of 2,346 eligible participants, $904(38.5 \%)$ refused, and $1,442(61.5 \%)$ attended our clinical testing protocol, giving an initial response rate of $61.5 \%$. Of these, $1,170(81 \%)$ were cognitively able to complete the questionnaire assessment. The mean age $\pm \mathrm{SD}$ of our participants was 73.8 \pm 8.6 years; $n=798(55.3 \%)$ were female; and $828(57.4 \%)$ were of Chinese ethnicity. The findings from this study will allow a deeper understanding of the risk factors and impact of aging in Asian populations, particularly in relation to the visual function and other functional system.
\end{abstract}

Key words: epidemiology, aging, eye disease, visual impairment, visual function system, population-based study

Population ageing is a global phenomenon, with 1.5 billion people estimated to be aged $\geq 60$ years worldwide by 2030 [1]. However, $60 \%$ of these people will be living in Asia [2]. Globally, Singapore has the world's highest *Correspondence should be addressed to: Dr. Ecosse L. Lamoureux, Singapore Eye Research Institute (SERI), Singapore. Email:
ecosse.lamoureux@ seri.com.sg.

Copyright: ( 2020 Gupta P et al. This is an open-access article distributed under the terms of the Creative Commons Attribution License, which permits unrestricted use, distribution, and reproduction in any medium, provided the original author and source are credited. 
average life expectancy at 85 years, and 1 in 4 people will be aged over 65 years within the next two decades [3-5]. This ageing phenomenon is expected to lead to a corresponding increase in the number of older people in Singapore living with age-related eye disorders, as the prevalence of these diseases increases dramatically after 70 years of age [6].

The exact impact of aging on the prevalence of eye diseases in Asian societies is unclear. Although several Asian population-based studies [7-21] have provided information on the epidemiology of eye diseases, most have recruited "middle-aged (40-50 years)" and "youngold (55-65 years)" participants, with a lack of information in those aged $>75$ years. For example, our Singapore Epidemiology of Eye Diseases (SEED) Study, had $<10 \%$ of its participants aged $>75$ years [22, 23]. Furthermore, previous studies have primarily focussed on measuring visual acuity (VA), with little information on other components of the visual function system such as contrast sensitivity [CS], stereo-acuity [SA], colour vision [CV], and field of view, which are strongly age-related and play critical roles in independence, navigation in the community, activities of daily living and quality of life (QoL) [24-26]. Moreover, while previous epidemiological studies have been 'eye-centric', the ageing of the eye does not happen in isolation. For instance, age-related eye diseases occur concurrently with other comorbidities and declines in other functional systems [27]. Therefore, a more holistic understanding of the deterioration in the components of the ageing visual system, in conjunction with age-related changes in other functional systems such as body composition, sensory, dental, and bone health, in elderly Singaporeans is imperative to inform public health policy and resource allocation planning. Unfortunately, such data are currently unavailable in Asian populations.

To bridge this knowledge gap, we implemented a nationally representative and population-based study of Chinese, Malays, and Indians aged $\geq 60$ years, entitled "The PopulatION HEalth and Eye Disease PRofilE in Elderly Singaporeans (PIONEER)." PIONEER aims to: (a) determine the epidemiology of age-related ocular pathologies, vision impairment (VI), and associated changes in various components of the ageing visual function system (primary outcome); (b) evaluate the cross-sectional and longitudinal relationships between age-related eye diseases, VI, the ageing visual function system and nutrition, other sensory losses (hearing and smell), oral health, cognition, objective body composition indices and multimorbidity; and (c) elicit the comprehensive economic, clinical and patientcentred impact of age-related eye diseases, VI and the ageing visual function system on the individual and caregiver.
In this paper, we provide a detailed description of the methodology of the PIONEER study.

\section{MATERIALS AND METHODS}

\section{Study Design and population}

PIONEER is currently a cross-sectional, population-based and epidemiological study of Chinese, Malay and Indian adults who are Singapore citizens or permanent residents aged 60 years and older living across Singapore. The study is intended to be longitudinal, with several waves of data planned to be collected in the future. The study commenced in December 2017 and follows the principles of the Declaration of Helsinki, with ethics approval obtained from the SingHealth Centralized Institutional Review Board (CIRB, Reference \#2016/3089). All participants are given a choice to provide their written, informed consent in either Chinese, Malay, Tamil or English. The study protocol and rationale are explained to participants in their preferred language including, common Singaporean dialects such as Hokkien, Cantonese, Teochew and Hakka, as well as common Indian languages, including Hindi, Punjabi etc. by trained study coordinators proficient in these languages/dialects.

PIONEER assessment comprises two sessions including (1) a comprehensive clinical examination conducted at Singapore Eye Research Institute (SERI), or on a mobile eye clinic, a custom built bus outfitted with standardized clinic equipment; and (2) an extensive questionnaire protocol subsequently administered at the participant's home in his/her preferred language, by trained interviewers. The cognitive status of the participants is assessed using the 6 item Cognitive Impairment Test (6-CIT) [28] before proceeding with the study procedures. For those who score $\geqq 8$ in 6-CIT (indicating mild cognitive impairment or worse) during the recruitment visit, the study details are explained to, and verbal consent obtained from, the primary caregiver. Furthermore, on the day of the clinical examination, research coordinators made sure to obtain written consent from the caregiver in the presence of an impartial witness, before commencing study related procedures. A copy of the signed consent form was given to the participant/caregiver per our study protocol. Those with 6-CIT scores $<8$ (no cognitive impairment) undergo the full (clinical and questionnaire assessment) protocol, whilst individuals with cognitive impairment undergo only the clinical assessment.

\section{Sampling Frame}

Of the 5.8 million resident population in Singapore, ethnic Chinese (74.4\%), Malays (13.4\%) and Indians (9.0\%) 
make up the majority of the population. Given that other ethnic groups (Eurasians, Caucasians, Japanese, Filipino and Vietnamese) contribute to only $\sim 3.2 \%$ of its population, we chose to focus only the three main ethnic groups in PIONEER. We used criteria set by the Singapore census to define 'Chinese', 'Malay' and 'Indian' ethnicities. This definition includes all persons of Chinese, Malay or Indian origin, as indicated on the National Registration Identity Card, which is provided to all Singapore citizens and permanent residents. We have used this definition in our previous population-based studies in Singapore [29-31].
In 2017, a geographically representative sample of 7000 Singaporeans aged $\geq 60$ years (stratified by age, gender, and ethnicity, and based on the estimated 2016 population distribution) residing across Singapore was obtained from a national database which have been used in previous population-based studies [32], to participate in the PIONEER Study. Individuals of Malay and Indian ethnicity, females, and older age groups were oversampled to ensure enough respondents for analyses in these groups.

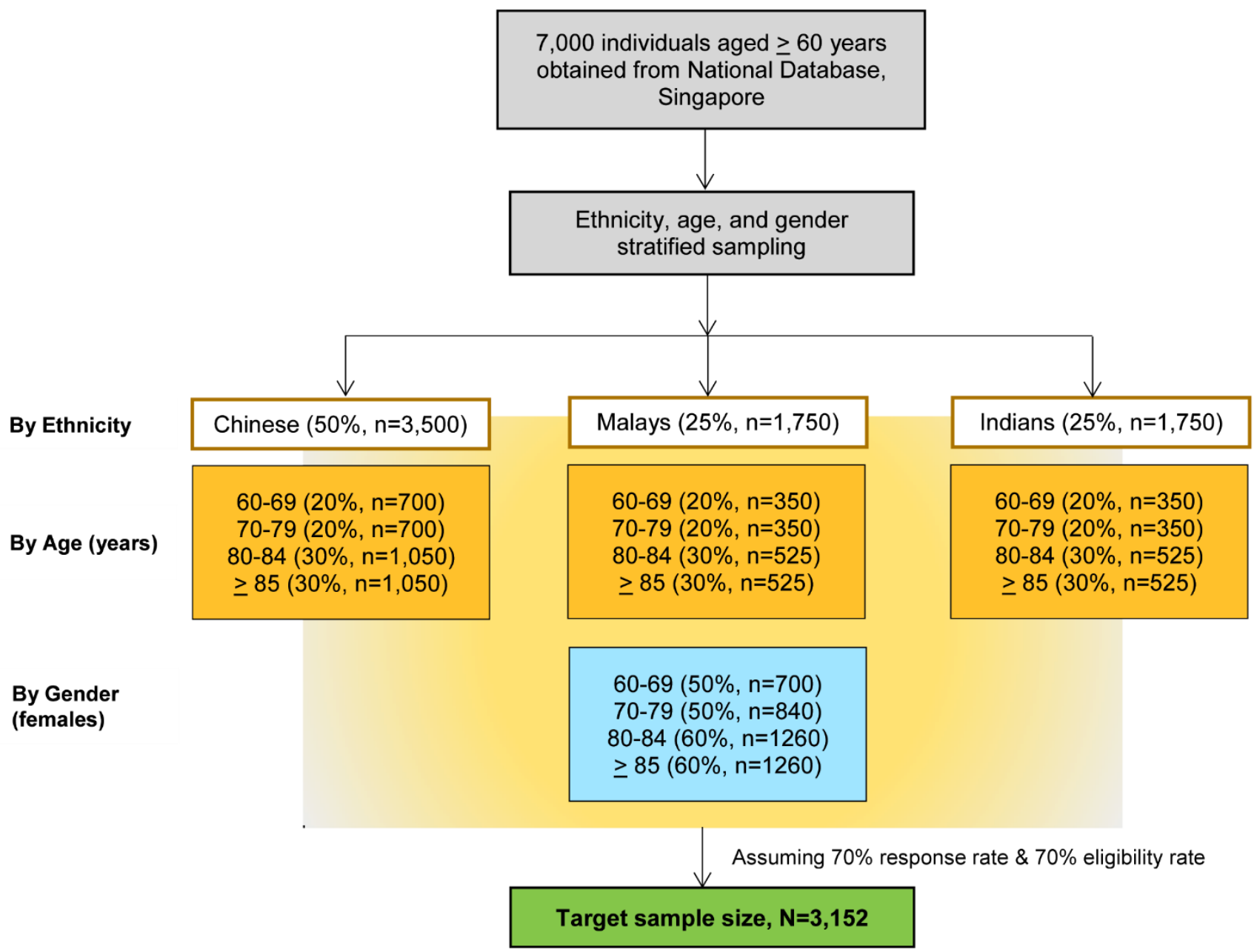

Figure 1. PIONEER sampling strategy and target sample size.

\section{Sample Size and Power}

The sample size needed to estimate the prevalence of VI and major age-related eye diseases (cataract, refractive error, presbyopia, age related macular degeneration, glaucoma, diabetic retinopathy) - with $95 \%$ confidence intervals not wider than $4 \%$ ranged from $\mathrm{N}=707$ for glaucoma to N=1936 for VI. Prevalence estimates were obtained from an analysis of participants aged above 70 years from a local population-based study, the Singapore Epidemiology of Eye Diseases study [33]. To have an adequately powered sample for subgroup analyses involving stratification by age, gender, ethnicity and visual function components (CS, SA, and CV), we 
increased the target sample size from 1936 to 3152 individuals. This sample size is adequate for estimating the prevalence of our secondary outcomes such as hearing loss, mobility, falls, cognition, sarcopenia, frailty, and independent living with the same level of precision as our primary outcomes. Assuming an eligibility rate of $70 \%$ and a response rate of $70 \%$, we estimated that a sampling frame of 7000 names would be sufficient to achieve our target sample size.

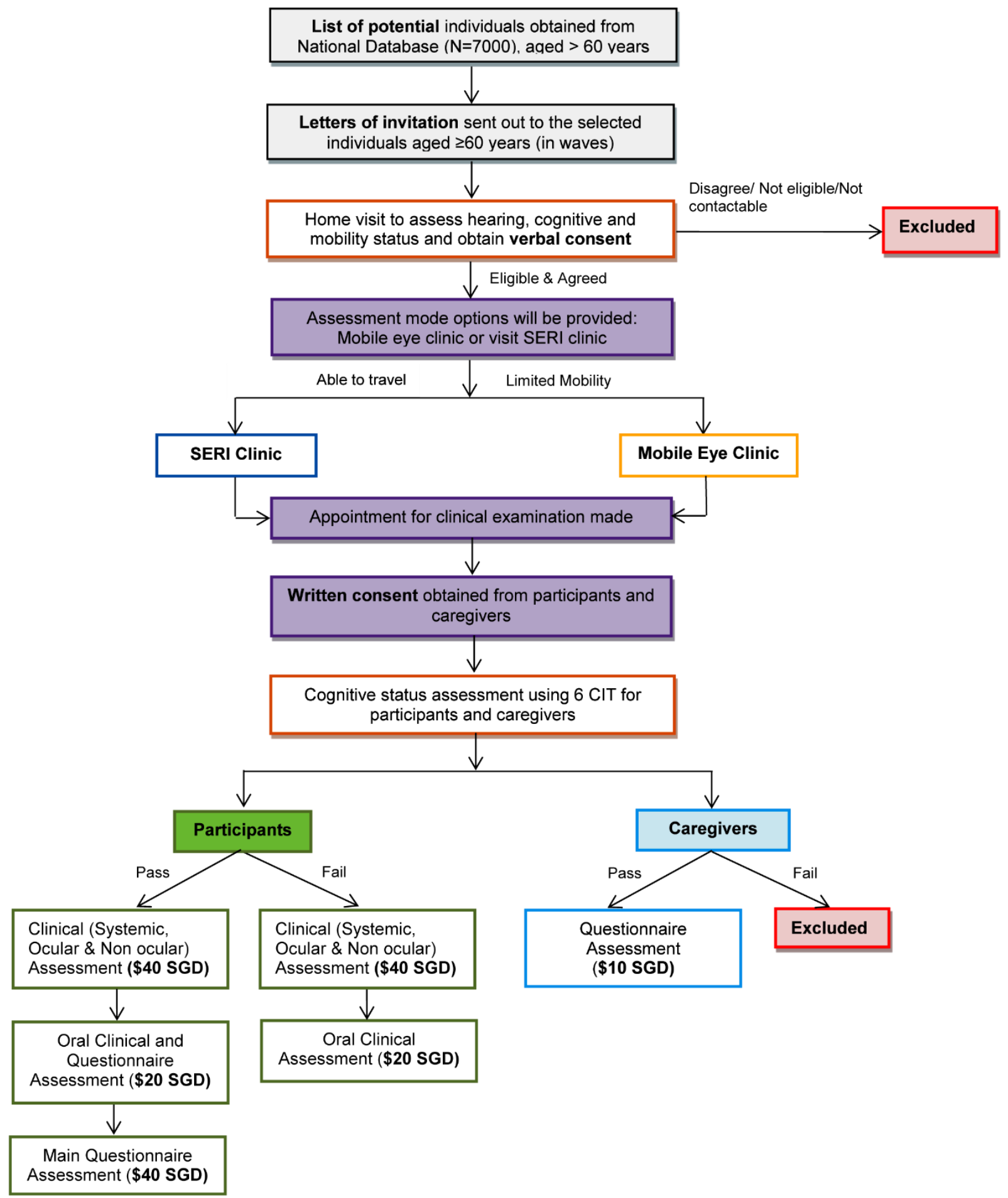

Figure 2. PIONEER study protocol. 
We performed a single-stage stratified random sampling of individuals based on ethnicity sampling fractions of $50 \%, 25 \%$ and $25 \%$ for Chinese, Malays and Indians, respectively, i.e. 3500, 1750, 1750. Within each ethnic group, sampling fractions were $20 \%, 20 \%, 30 \%$ and $30 \%$ for the age groups $60-69 y, 70-79 y, 80-84 y$, and $\geq 85 \mathrm{y}$, respectively $(700,700,1050,1050$ for the four age groups in Chinese; 350, 350, 525, and 525 in Malays and Indians). Lastly, female sampling fractions were $50 \%$, $50 \%, 60 \%$, and $60 \%$ for the age groups $60-69 y, 70-79 y$, $80-84 \mathrm{y}$, and $\geq 85 \mathrm{y}$, respectively. The corresponding estimates for the final sample size of 3152 stratified by ethnicity, gender and age are shown in Figure 1.

We over sampled minority ethnic groups (Malays and Indians), females, and older age groups, to increase the precision of estimates in these smaller subgroups. As such disproportionate sampling skews the distribution of the overall sample in terms of age, gender and ethnicity from the national distribution, individuals have to be weighted according to the resulting probabilities of being selected so that aggregated estimates are calibrated in line with the national distribution in terms of age, gender and ethnicity. For example, more weight will be given to males since their selection probabilities have been reduced relative to females, while less weight will be given to Malays and Indians as their selection probabilities have been inflated relative to Chinese when sampling from the national pool. We will define these sampling weights using the statistical method of post-stratification by aligning age, gender and ethnicity distribution to that of the national sampling frame. Similarly, not all sampled individuals will eventually participate, and participants may differ systematically from non-participants in terms of the distribution of age, gender and ethnicity (we will unfortunately not be able to collect other information on non-participants as we had to comply with Singapore's Personal Data Protection (privacy) Act). Depending on how large these differences come to be, we will also consider applying weights for non-response following the same principles for calculating sampling weights as described above.

\section{Eligibility Criteria and Recruitment Strategies}

All 7000 names are initially considered eligible to participate. Therefore, an invitation letter outlining the study details and inviting the addressee and caregiver to participate in the study is sent to all individuals on the list, in batches. This is followed by a visit to their home by the study recruitment officers to ascertain eligibility and agreement to participate. A potential participant is considered "ineligible" if he/she is uncontactable, in prison, residing in nursing home, deceased or terminally ill, bedridden or otherwise unable to give informed consent (e.g. due to dementia, severe hearing impairment, or muteness). Following an on-site eligibility assessment by the recruitment officer of a potential participant's hearing (self-reported), cognitive (6-item Cognitive Impairment Test) [28], and mobility (self-reported) status, all eligible individuals are provided with printed and verbal information on the examination procedures and associated benefits and risks. Thereafter, if the individual is interested, verbal consent is obtained, and a clinic appointment is made. If the participant is absent during the home visit, a letter is left to encourage him/her to contact the study team. A potential participant is termed "not contactable" after six unsuccessful telephone calls and four home visits each conducted at different days (including weekdays and weekends) and times (morning, afternoon and evening). Individuals on the sampling list who declined the initial invitation are contacted again at a later time in a further attempt to recruit him/her, with at least one contact made every 3 months. The PIONEER recruitment strategies are shown in Figure 2.

For those who agree to participate, a reminder telephone call is made a day prior to the scheduled appointment to remind participants to attend. An individual who misses his/her appointment will have the appointment rescheduled. On the day of their appointment, study participants are requested to bring along their national identity card, and habitual distance and near spectacles. In addition, they are advised to refrain from wearing contact lenses, if worn, in order to avoid potential distortions and aberrations of the cornea arising from contact lens wear. As part of the informed consent process, our recruitment and study officers are trained to ensure all potential participants are aware that participation in the study is entirely voluntary and that they have the right to withdraw from the study or any specific clinical assessments at any time. Our clinical testing protocol takes on average $\sim 3.5$ hours. In cases where our elderly participants are unable to complete the tests during the same visit, another appointment is made within 1 month from the first visit with the participant's consent, to complete all the remaining clinical assessments, along with the questionnaire administration. Furthermore, we offer free transportation and pick-up services to our subjects, and following the successful completion of the assessments, the participant is reimbursed for his/her time.

Regardless of the cognitive status of individuals who agree to participate, their primary caregiver is also contacted to ask for his/her willingness to be interviewed to assess the quality of life impact of caring for elderly relatives from the carer's perspective. Caregivers also complete the 6-CIT to assess cognitive functioning before written informed consent is taken, with those who fail being excluded from this portion of the study. 


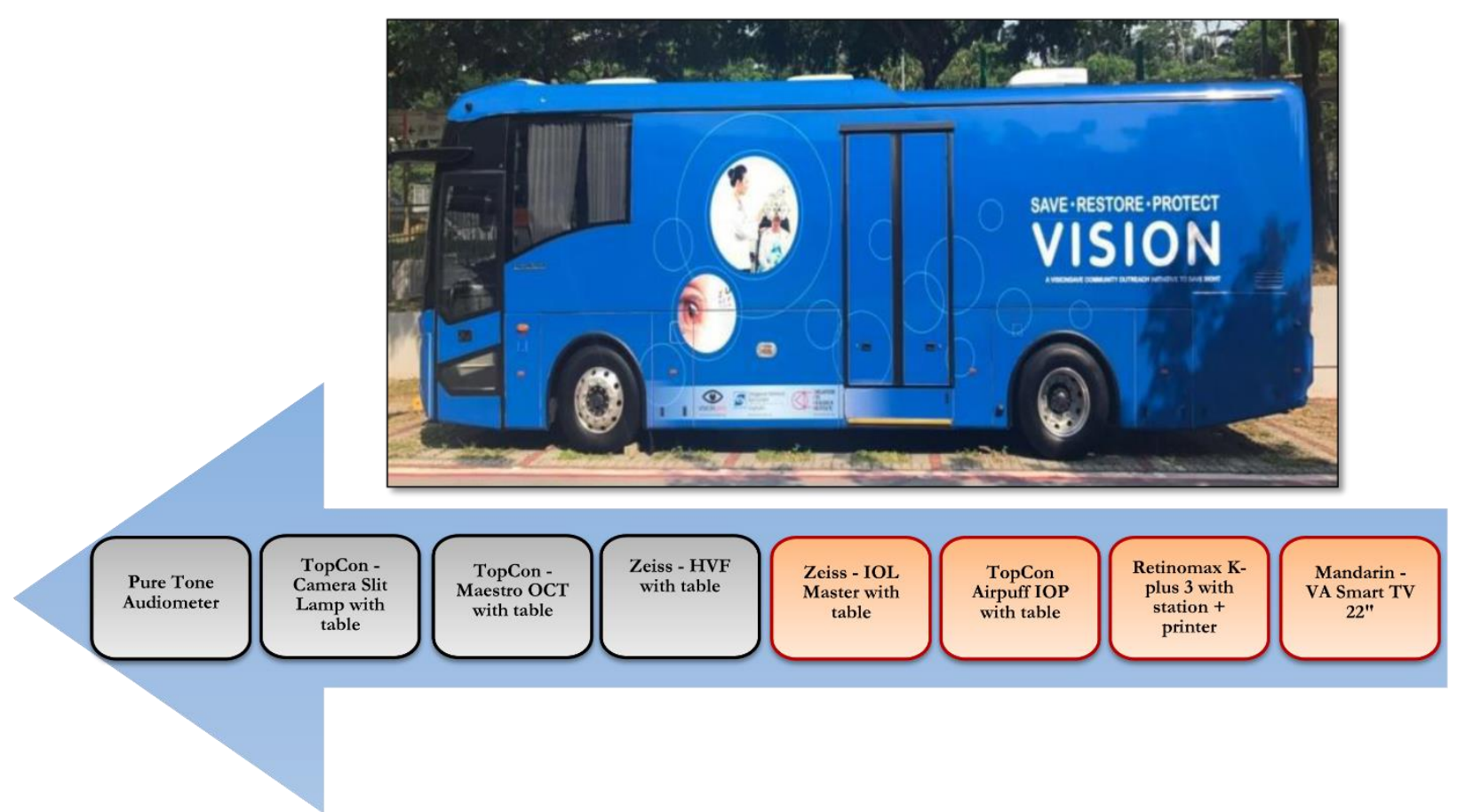

Figure 3. A custom-designed mobile eye clinic, equipped with all necessary equipments to undertake the study protocol for participants with limited mobility.

\section{Clinical Examination (systemic, ocular, and non-ocular)}

The clinical assessment component of PIONEER is conducted at SERI. For those participants with limited mobility (e.g. wheelchair bound), we offer the option to undergo study testing in a custom-designed mobile eye clinic, equipped with all the necessary equipments to undertake the study protocol (Fig. 3). At the study clinic, participants undergo a comprehensive battery of standardised examination procedures (Fig. 4), including:

- Physical (height, weight, waist and hip circumference, body mass index-BMI, and blood pressure-BP)

- Ocular (visual function assessment including VA, CS, SA, CV, standard visual fields and attentional field of view, slit lamp, gonioscopy, pupil dilation, fundus photography and optical coherence tomography imaging)

- Non-ocular (gait speed, grip strength, body composition and bone health, physical activity, frailty, hearing, smell identification, and dental assessment)

- Laboratory components (non-fasting venous blood and urine sample)

\section{Assessment of the Components of the Visual Function System}

Distance Visual Acuity (presenting \& best corrected) and subjective refraction: Monocular and binocular presenting distance visual acuities (PDVA) are assessed using a LogMAR (Log of Minimum Angle of Resolution) chart (Lighthouse International, Distance VA Number Chart, CAT No. C102) with the participant wearing his/her habitual prescription under photopic conditions $\left(85 \mathrm{~cd} / \mathrm{m}^{2}\right)$ at $4 \mathrm{~m}$. If the participants are unable to read the largest line of letters on the VA chart, the chart is moved to $2 \mathrm{~m}$. However, if he/she is still unable to make out any lines at $2 \mathrm{~m}$, finger counting, hand movement and the ability of the eye to perceive light with a pen torch are assessed. Binocular measurements are made with both eyes viewing the target. Monocular and binocular best corrected distance VA (BCDVA) is then assessed using a trial frame and lenses under the same photopic conditions as that of PDVA measurements. Using the auto-refraction results as a starting point, the refinement of the sphere, cylinder and axis is performed until the BCDVA is obtained. Both PDVA and BCDVA are scored on a letter by letter basis with each letter worth $0.02 \log$ units.

Near Visual Acuity: Binocular presenting near VA (PNVA) is performed under photopic conditions at $40 \mathrm{~cm}$ with the participant's habitual correction. The same PDVA and BCDVA methodology is used to assess PNVA, namely the last line attempted where $\geq 3$ mistakes are made, combined with the number of mistakes made on previous lines, are used to calculate a letter-by-letter $\log$ MAR PNVA score. Binocular best corrected near VA (BCNVA) measurement is conducted after BCDVA 
assessment, with participants' best corrected prescription for the testing distance. Plus sphere trial lenses are utilized to obtain BCNVA measurements binocularly. The spherical dioptric correction is recorded along with the corresponding BCNVA.

Contrast Sensitivity (CS) (ability to recognise targets of different levels of contrast [faintness]) is measured using Pelli-Robson Contrast Sensitivity Chart as described elsewhere [34]. In brief, CS is measured monocularly and binocularly with the participant's best distance correction, corrected for the testing distance of 1 $\mathrm{m}$ with a $+0.75 \mathrm{D}$ working distance lens under photopic condition. Scores range from 0.00 to $2.25 \log C S$ with higher values indicating better CS.
Stereo-Acuity (ability to see in 3-D) is assessed using the Frisby Stereo test [35]. Participants are presented with a stereo image on a sequence of three transparent plates, and are asked to identify the circle that has the depth cue in one of four squares for each of the three plates at a distance of $60 \mathrm{~cm}$ while wearing best corrected near correction. Scores range from 40-150 arc sec with lower values indicating better SA.

Colour Vision is measured using the Farnsworth D15 test. Participants are asked to arrange 15 different colour discs in a sequential colour series, monocularly, while wearing best corrected near correction. Abnormal colour vision is defined as one or more major crossings, where the difference between two adjacent caps is more than three steps in the better-eye.

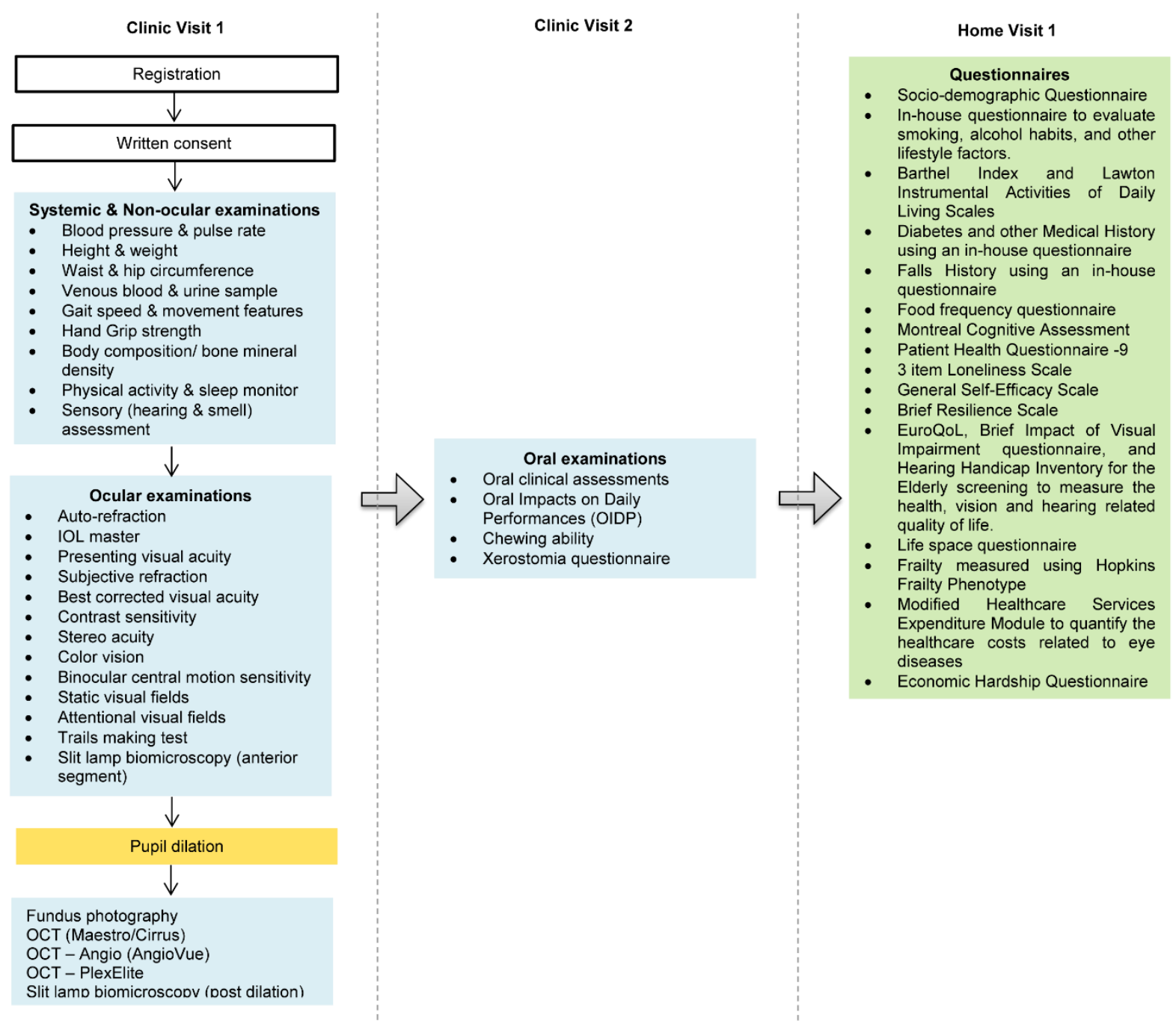

Figure 4. PIONEER battery of clinical and questionnaire assessments.

Other Ocular Assessments

Binocular Central Motion Sensitivity: Motion sensitivity is assessed binocularly with habitual distance correction at a $3.2 \mathrm{~m}$ testing distance. Participants report the perceived direction of movement of a group of small targets generated on a computer screen (which move in one of four directions) and the minimum amount of 
stimulus movement that is correctly perceived is recorded as a displacement threshold, in logDegArc.

Attentional Field of View (AFoV): AFoV is a functional test of binocular visual processing speed for rapid detection and localization of central and peripheral targets under conditions of divided and selective visual attention (absence and presence of visual distractors, respectively). The test assesses higher order cognitive abilities, but performance also relies on visual sensory function since targets must be visible in order to be detected. The test has demonstrated high levels of reliability and validity $[36,37]$. The test is conducted on an iPad, where participants identify a central task (direction of a large high-contrast tumbling E) and simultaneously identify the location of a peripheral target (a low-contrast Gabor patch). Processing speeds are calculated as the minimum presentation time (in ms) at which participants correctly identify both the central and peripheral targets. Faster speeds indicate better visual processing speeds.

Neuro-psychological test of Visual Attention Trail Making Test (TMT): Trail-making A and B tests are pen and paper tests which participants complete binocularly with their habitual near correction. In Part A, participants are asked to draw lines to connect in ascending order a series of randomly positioned circles containing numbers $(1-2-3 . .25)$, as quickly as possible. In Part B, the circles include both numbers and letters, and participants are required to draw lines to connect the circles in an alternating ascending order between the numbers and letters (1-A-2-B-..13-L), which requires additional executive function processing during the visual search task. The time to complete both tests is recorded in seconds.

\section{Non-ocular Assessments}

Gait speed is assessed with participants walking a distance of $4 \mathrm{~m}$ (15 feet) at their usual speed. Scoring of the gait speed test is in seconds. A score of less than 0.8 $\mathrm{m} / \mathrm{s}$ is defined as slow gait speed [38].

Gait features using ZurichMOVE Sensor: The ZurichMOVE Sensor provides gait parameters during a typical walking session, including walking speed $(\mathrm{m} / \mathrm{sec})$, dual limb support time (sec; the duration of time when both feet are in contact with the ground during walking), stride time (sec; the duration between consecutive strides), stride length $(\mathrm{m}$; the distance between consecutive strides), step time (sec), step length (m), and minimum toe clearance $(\mathrm{m}$; the minimum distance with respect to the ground during the swing phase of gait) [39]. Participants are asked to walk for a minimum of 2.5 minutes to a maximum of 6 minutes. The variability of dual limb support time is linked to balance control, while variability in stride time provides rhythmicity. Similarly, variability of step length and time provide coordination and variability in minimum toe clearance is associated with obstacle avoidance [40-42]. The variability in these parameters over the entire period of walking will provide the gait features.

Hand Grip Strength: Participants' grip strength (in kilograms) in the dominant hand is measured three times, with the participant seated and elbow flexed at 90 degrees, with a rest period of 30 seconds between each measurement. The average of the three readings will be utilised in analyses.

Objective Physical Activity and Sleep Monitor: Physical activity and sleep are monitored using an ActiGraph accelerometer (GT9X band). Participants are instructed to wear the watch on the non-dominant wrist over a period of 7 consecutive days ( 24 hours a day, except during water-based activities) and maintain a sleep $\log$. The watch is collected by the study coordinator on the $8^{\text {th }}$ day. Raw acceleration data, number of steps taken, physical activity intensity (mild, moderate or vigorous), sleep latency, total sleep time and sleeping efficiency data are retrieved from the watch.

Body Composition \& Bone Mineral Density measures are assessed using dual energy X-ray absorptiometry (DXA; Hologic Discovery-W; Hologic Inc, BedfordMA), which is a non-invasive imaging modality utilizing very low dose $\mathrm{X}$-rays ( 0.005 to 0.01 millisievert). DXA quantifies participants' body composition profiles, including fat and muscle mass, and bone mineral density (for entire single hip, and lumbar spine) measures. Standard patient preparation and procedural protocol are followed. The examinations are performed by an Allied Health Professions Council accredited radiographer to ensure accuracy in positioning and delineation of bone map and region of interest.

A detailed description of the clinical protocol, and definitions of ocular and systemic diseases are shown in Supplementary Table 1 .

\section{Questionnaire Administration}

During home interviews, all questionnaires are digitally administered using a tablet to participants who pass the 6CIT in order to collect data on the following outcomes:

- Demographic (age, gender, marital status)

- Socio-economic (education, family income, housing, occupation)

- Lifestyle (smoking, alcohol consumption, physical activity)

- Self-reported history of systemic and eye diseases, medication use, and surgical history

- Family history of systemic and eye diseases 
- History of falls and fractures

- Cognitive function [43]

- Depression [44]

- Loneliness, social isolation [45], and behaviour (selfefficacy) [46], [47]

- General health [48], vision [49], and hearing related QoL [50]

- Independence (activities of daily living [ADL] [51], instrumental activities of daily living [IADL]) [52]

- Frailty [53]

- Nutrition [54]

- Economic impact [55] and household economic hardship [56]

- Oral health [57]

- Adult carer QoL [58]

A detailed description of the questionnaire protocol is provided in Supplementary Table 2. All questionnaires capturing patient reported outcomes, including, cognition [59], depression [60], functional status (ADL, IADL) [61, 62], social isolation [63], quality of life (general, vision and hearing related) [64-66], frailty [67] and oral health [57] have been previously validated in our local population studies.

\section{Quality Assurance and Control}

All study officers including interviewers and recruitment officers are required to undertake Research Ethics and Compliance Training (an online program for research ethics and compliance education). They are trained by qualified personnel and are required to demonstrate competency in the relevant procedures and questionnaire administration before being certified to perform these procedures and conduct interviews on participants. Study informed consent and all questionnaires are translated into Mandarin, Malay and Tamil languages, and then back translated into English, by a certified translation company. Furthermore, the translated versions are proofread to ensure the meaning is consistent with the original. A detailed checklist outlining the study inclusion/exclusion criteria is given to the recruitment officers to conduct proper eligibility testing. A pilot study $(\mathrm{N}=20 ; 5$ of each language) was conducted prior to commencement of the main study to test out PIONEER recruitment strategies and workflow and ensure that all staff are familiar with the examination procedures. The quality of the data collected is checked periodically by the key investigators. Quality control test for BMD is performed everyday using Hologic Anthropomorphic Spine Phantom. Automatic Body Composition Calibration and radiographic uniformity test are done on a weekly basis. All study instruments are serviced annually (or as specified) to ensure accuracy in measurements.

\section{Data Storage}

Data are collected in paper and digital formats via the research electronic data capture software (REDCap, Singapore). Clinical examination records, questionnaire responses, printouts (e.g., autorefraction, intra-ocular lens [IOL] master parameters), and biochemistry results are compiled into participant-specific case report forms that are labeled with the participant's unique study number. Imaging data, including digital fundus, retinal imaging, and DXA images and analyses report are retrieved directly from the imaging equipment and stored in their respective computers, identifiable only by the study number, date created, file path, format, and size. The password protected database is sent to a dedicated data management team for implementation of data management procedures including quality checks and further data cleaning. Retinal photographs are stored in digital format for grading. Images are backed up on to the external drive and then securely transferred on to the SERI server. This storage method ensures there are two sets of copies in different formats. Finally, monthly meetings are conducted between data collection staff, the project manager (PG) and the principal investigator (EL) to discuss discrepancies identified during data collection.

\section{Statistical Analyses}

Analyses will be performed using the commercially available STATA statistical software (Version 15, StataCorp, College Station, Texas). In future analyses, age- and gender-standardized prevalence rates will be calculated for major age-related eye diseases, VI, low vision, and presbyopia. Regression analyses under the generalized linear models' framework will be used to:

1) Compare visual system components between those with and without specific eye diseases adjusted for traditional risk factors (i.e. hypertension, diabetes, cardiovascular disease).

2) Determine the associations between multimorbidity, multimorbidity-related factors (such as multiple treatment modalities, polypharmacy and the burden of disease management such as multiple daily drops for glaucoma and monthly injections for retinal diseases) with prevalent eye diseases and components of the ageing function system. A summary multimorbidity score will be created based on totaling the number of comorbid conditions and analyzed both as a continuous score and as a categorical variable ( 0 $1,2-4, \geq 5)$. 
3) Determine the associations between body composition measures (e.g. fat and muscle mass) with age-related eye diseases.

4) Determine the associations between major eye diseases and VI, and cognitive impairment, psychosocial wellbeing, and vision specific QoL data.

Where eye-specific analyses are concerned, the approach of generalized estimating equations will be used to estimate marginal associations that take into consideration the correlation between the two eyes of an individual. The economic impact of eye diseases, VI and the ageing visual function system, will be assessed by separately presenting estimates of direct and indirect costs by key stratification variables, including age, gender and ethnicity.

\section{RESULTS}

A total of 3,299 participants (from East, West, North and South Singapore) were approached from December 2017 to November 2019. Of these, 953 (28.5\%) were deemed ineligible. Out of 2,346 eligible participants, 904 (38.5\%) refused, and 1,442 $(61.5 \%)$ attended our clinical testing protocol, giving an initial response rate of $61.5 \%$. Of these, $1,170(81 \%)$ were cognitively able to complete the questionnaire assessment.

Table 1. Age, gender and ethnicity of participants $(n=1442)$ vs. non-participants $(n=904)$.

\begin{tabular}{lccc}
\hline \multicolumn{4}{c}{ Mean \pm SD or n (\%) } \\
\hline & $\begin{array}{c}\text { Participants } \\
\mathbf{n = 1 4 4 2}\end{array}$ & $\begin{array}{c}\text { Non-participants } \\
\mathbf{n}=\mathbf{9 0 4}\end{array}$ & $\mathbf{P}$ \\
\hline Age & $73.8 \pm 8.6$ & $77.6 \pm 8.7$ & $<0.001$ \\
Gender & & & \\
Male & $642(44.5 \%)$ & $357(39.5 \%)$ & 0.015 \\
Female & $798(55.3 \%)$ & $547(60.5 \%)$ & \\
Ethnicity & & & \\
Chinese & $828(57.4 \%)$ & $673(74.5 \%)$ & $<0.001$ \\
Indian & $420(29.1 \%)$ & $165(18.3 \%)$ & \\
Malay & $160(11.1 \%)$ & $66(7.3 \%)$ & \\
\hline
\end{tabular}

A substantial proportion of our sample was deemed ineligible to participate in the study. This was because our sampling frame only contained the individual's name, age, gender, ethnicity, contact address and his/her regional residential zone. Participants' cognitive, hearing and mobility status, and other health conditions (e.g., bedridden) were ascertained only at the home visit screening. Likewise, the status of participants residing in nursing home or prison was only discovered after the home visit screening.

Table 1 compares the age, gender and ethnicity data of non-participants $(n=904)$ versus participants $(n=1442)$. Compared to participants, non-participants were older, more likely to be female and Chinese. However, we could not compare other sociodemographic data of nonrespondents with respondents in order to comply with Singapore's Personal Data Protection (privacy) Act.

\section{DISCUSSION}

PIONEER is an elderly- and systemic-centric, populationbased study of Chinese, Indians and Malays. The study is ideally placed to investigate the clinical, biological, anthropometric, and psychosocial phenotypes of contemporary elderly Singaporeans living with vision loss and other sensory impairments to better understand the magnitude, consequences, burden, and complex mechanisms underlying ageing. Upon completion and with information from all ethnic groups, PIONEER will likely contribute to new and much needed data of the burden associated with age-related sensory decline in Singapore to inform public health initiatives; and assist policy and decision makers in planning health care availability, utilization, and resource allocation. Moreover, PIONEER will likely inform researchers and clinicians about the economic burden and QoL impact associated specifically with the ageing visual function system as well as multimorbidity more generally.

The main strengths of PIONEER include its extremely well-characterized participant sample, with high-quality ocular and systemic data, and a rich collection of bio-samples. The examinations in PIONEER are standardised and reproducible with a strong emphasis on quality control. Use of standardised protocols will enable direct comparison, validation, and pooling of analyses with other population-based studies. Furthermore, PIONEER focuses exclusively on older participants ( $\geq 60$ years), a sector of our population that has not been well studied previously and will thus offer novel insights into the world of an elderly individual. The current study has some limitations. First, our detailed clinical testing protocol of $\sim 3.5$ hours resulted in fatigue in some of our elderly participants. However, it is unlikely to affect our data quality as we followed a randomized testing order and our participants were given appropriate breaks during the testing process. Moreover, we have split our clinical protocol into two visits for subjects who cannot manage the long testing protocol. Second, as the questionnaire protocol was only administered to the participants who passed the 6-CIT, we do not have patient-reported outcomes data on cognitively impaired individuals. Third, our initial recruitment rate is slightly 
low $(\sim 61.5 \%)$. This is likely because telephone numbers for our participants were not available (due to the Personal Data Protection Act regulation in Singapore), resulting in home-based (as opposed to telephone-based) recruitment, which is an extremely time consuming and inefficient recruitment method. However, we have adopted several measures to improve the participation rate of our study, such as increasing the number of recruitment officers, offering free transportation and pick-up services to our subjects, and providing printed information (such as brochures) on the study procedures and their associated benefits and risks at the point of recruitment. The current response rate is $\sim 65 \%$.

In summary, PIONEER employs a methodical elderly- and systemic-centric approach to provide novel insights into how the visual function system changes with age, its interaction with nutrition, sensory measures, oral, mental, and bone health, body composition and multimorbidity; as well as the consequences and burden of these multi-morbid visual changes. Furthermore, PIONEER comprehensively evaluates the impact of eye diseases, VI, and ageing visual function from a patientcentred perspective, supply information on the burden of eye disease and multimorbidity from the caregiver's perspective and quantify the societal costs and personal economic hardships associated with VI. Such data will generate an evidence-based cost-effectiveness model needed to assess the economic benefits of interventions and treatment modalities in this segment of the Singapore population. Information from this study will be critical for the planning of public health strategies for screening, early diagnosis and intervention in various ocular and systemic diseases, translatable not only to Singaporeans but to other Asian populations outside Singapore.

\section{Acknowledgements}

The study was supported by grant from Singapore National Medical Research Council (NMRC-CSA-SI \#JRNMRR140601).

\section{Conflict of Interest}

All authors have completed and submitted the ICJME Forms for Disclosure of Potential Conflicts of Interest and none were reported.

\section{Supplementary Materials}

The Supplemenantry data can be found online at: www.aginganddisease.org/EN/10.14336/AD.2020.0206.

\section{References}

[1] Vaupel JW (2010). Biodemography of human ageing. Nature, 464:536-542.

[2] Beard JR, Officer A, de Carvalho IA, Sadana R, Pot AM, Michel JP, et al. (2016). The World report on ageing and health: a policy framework for healthy ageing. Lancet, 387:2145-2154.

[3] Choo PW, Lee KS, Owen RE, Jayaratnam FJ (1990). Singapore--an ageing society. Singapore Med J, 31:486-488.

[4] Rogerson A, Stacey S (2018). Successful Ageing in Singapore. Geriatrics (Basel), 3.

[5] Bai R, Wei J, An R, Li Y, Collett L, Dang S, et al. (2018). Trends in Life Expectancy and Its Association with Economic Factors in the Belt and Road Countries-Evidence from 2000(-)2014. Int J Environ Res Public Health, 15.

[6] Chader GJ, Taylor A (2013). Preface: The aging eye: normal changes, age-related diseases, and sight-saving approaches. Invest Ophthalmol Vis Sci, 54:ORSF1-4.

[7] Hsu WM, Cheng CY, Liu JH, Tsai SY, Chou P (2004). Prevalence and causes of visual impairment in an elderly Chinese population in Taiwan: the Shihpai Eye Study. Ophthalmology, 111:62-69.

[8] Nakamura Y, Tomidokoro A, Sawaguchi S, Sakai H, Iwase A, Araie M (2010). Prevalence and causes of low vision and blindness in a rural Southwest Island of Japan: the Kumejima study. Ophthalmology, 117:2315-2321.

[9] Liang YB, Friedman DS, Wong TY, Zhan SY, Sun LP, Wang JJ, et al. (2008). Prevalence and causes of low vision and blindness in a rural chinese adult population: the Handan Eye Study. Ophthalmology, 115:19651972.

[10] Dandona R, Dandona L, Naduvilath TJ, Nanda A, McCarty CA (1997). Design of a population-based study of visual impairment in India: The Andhra Pradesh Eye Disease Study. Indian J Ophthalmol, 45:251-257.

[11] Zhao J, Ellwein LB, Cui H, Ge J, Guan H, Lv J, et al. (2010). Prevalence of vision impairment in older adults in rural China: the China Nine-Province Survey. Ophthalmology, 117:409-416, 416 e401. Jonasson F, Arnarsson A, Sasaki H, Peto T, Sasaki K, Bird AC (2003). The prevalence of age-related maculopathy in iceland: Reykjavik eye study. Arch Ophthalmol, 121:379-385.

[13] Klaver CC, Wolfs RC, Vingerling JR, Hofman A, de Jong PT (1998). Age-specific prevalence and causes of blindness and visual impairment in an older population: the Rotterdam Study. Arch Ophthalmol, 116:653-658. Mitchell P, Smith W, Attebo K, Wang JJ (1995). Prevalence of age-related maculopathy in Australia. The Blue Mountains Eye Study. Ophthalmology, 102:1450-1460.

[15] Livingston PM, Carson CA, Stanislavsky YL, Lee SE, Guest CS, Taylor HR (1994). Methods for a population-based study of eye disease: the Melbourne Visual Impairment Project. Ophthalmic Epidemiol, 
1:139-148.

[16] Leske MC, Connell AM, Schachat AP, Hyman L (1994). The Barbados Eye Study. Prevalence of open angle glaucoma. Arch Ophthalmol, 112:821-829.

[17] Munoz B, West SK, Rodriguez J, Sanchez R, Broman AT, Snyder R, et al. (2002). Blindness, visual impairment and the problem of uncorrected refractive error in a Mexican-American population: Proyecto VER. Invest Ophthalmol Vis Sci, 43:608-614.

[18] Klein R, Klein BE, Linton KL, De Mets DL (1991). The Beaver Dam Eye Study: visual acuity. Ophthalmology, 98:1310-1315.

[19] Munoz B, West SK, Rubin GS, Schein OD, Quigley HA, Bressler SB, et al. (2000). Causes of blindness and visual impairment in a population of older Americans: The Salisbury Eye Evaluation Study. Arch Ophthalmol, 118:819-825.

[20] Varma R, Paz SH, Azen SP, Klein R, Globe D, Torres M, et al. (2004). The Los Angeles Latino Eye Study: design, methods, and baseline data. Ophthalmology, 111:1121-1131.

[21] Tielsch JM, Sommer A, Witt K, Katz J, Royall RM (1990). Blindness and visual impairment in an American urban population. The Baltimore Eye Survey. Arch Ophthalmol, 108:286-290.

[22] Foong AW, Saw SM, Loo JL, Shen S, Loon SC, Rosman M, et al. (2007). Rationale and methodology for a population-based study of eye diseases in Malay people: The Singapore Malay eye study (SiMES). Ophthalmic Epidemiol, 14:25-35.

[23] Lavanya R, Jeganathan VS, Zheng Y, Raju P, Cheung N, Tai ES, et al. (2009). Methodology of the Singapore Indian Chinese Cohort (SICC) eye study: quantifying ethnic variations in the epidemiology of eye diseases in Asians. Ophthalmic Epidemiol, 16:325-336.

[24] Haegerstrom-Portnoy G, Schneck ME, Brabyn JA (1999). Seeing into old age: vision function beyond acuity. Optom Vis Sci, 76:141-158.

[25] Owsley C, McGwin G, Jr., Sloane ME, Stalvey BT, Wells J (2001). Timed instrumental activities of daily living tasks: relationship to visual function in older adults. Optom Vis Sci, 78:350-359.

[26] Owsley C, McGwin G, Jr. (2004). Association between visual attention and mobility in older adults. J Am Geriatr Soc, 52:1901-1906.

[27] Kim JM, Kim SY, Chin HS, Kim HJ, Kim NR, Epidemiologic Survey Committee Of The Korean Ophthalmological Society O (2019). Relationships between Hearing Loss and the Prevalences of Cataract, Glaucoma, Diabetic Retinopathy, and Age-Related Macular Degeneration in Korea. J Clin Med, 8.

[28] Upadhyaya AK, Rajagopal M, Gale TM (2010). The Six Item Cognitive Impairment Test (6-CIT) as a screening test for dementia: comparison with MiniMental State Examination (MMSE). Curr Aging Sci, 3:138-142.

[29] Wong TY, Foster PJ, Seah SK, Chew PT (2000). Rates of hospital admissions for primary angle closure glaucoma among Chinese, Malays, and Indians in Singapore. Br J Ophthalmol, 84:990-992.

$[30]$
Wong TY (2001). Cataract extraction rates among Chinese, Malays, and Indians in Singapore: a population-based analysis. Arch Ophthalmol, 119:727-732.

Rosman M, Wong TY, Ong SG, Ang CL (2001). Retinal detachment in Chinese, Malay and Indian residents in Singapore: a comparative study on risk factors, clinical presentation and surgical outcomes. Int Ophthalmol, 24:101-106.

Cheng GH, Chan A, Ostbye T, Malhotra R (2019). Productive engagement patterns and their association with depressive symptomatology, loneliness, and cognitive function among older adults. Aging Ment

Health:1-9.
Saw SM, Foster PJ, Gazzard G, Seah S (2004). Causes of blindness, low vision, and questionnaire-assessed poor visual function in Singaporean Chinese adults: The Tanjong Pagar Survey. Ophthalmology, 111:11611168.

Wood JM, Anstey KJ, Kerr GK, Lacherez PF, Lord S (2008). A multidomain approach for predicting older driver safety under in-traffic road conditions. J Am Geriatr Soc, 56:986-993.

Frisby JP, Davis H, McMorrow K (1996). An improved training procedure as a precursor to testing young children with the Frisby Stereotest. Eye (Lond), 10 ( Pt 2):286-290.

Owsley C, Ball K, McGwin G, Sloane ME, Roenker DL, White MF, et al. (1998). Visual processing impairment and risk of motor vehicle crash among older adults. Journal of the American Medical Association, 279:1083-1088.

Ball KK, Roenker DL, Wadley VG, Edwards JD, Roth DL, McGwin G, Jr., et al. (2006). Can high-risk older drivers be identified through performance-based measures in a Department of Motor Vehicles setting? $\mathrm{J}$ Am Geriatr Soc, 54:77-84.

Chen LK, Liu LK, Woo J, Assantachai P, Auyeung TW, Bahyah KS, et al. (2014). Sarcopenia in Asia: consensus report of the Asian Working Group for Sarcopenia. J Am Med Dir Assoc, 15:95-101.

McGinnis RS, Mahadevan N, Moon Y, Seagers K, Sheth N, Wright JA, Jr., et al. (2017). A machine learning approach for gait speed estimation using skinmounted wearable sensors: From healthy controls to individuals with multiple sclerosis. PLoS One, 12:e0178366.

Hamacher D, Singh NB, Van Dieen JH, Heller MO, Taylor WR (2011). Kinematic measures for assessing gait stability in elderly individuals: a systematic review. J R Soc Interface, 8:1682-1698.

Konig N, Taylor WR, Baumann CR, Wenderoth N, Singh NB (2016). Revealing the quality of movement: A meta-analysis review to quantify the thresholds to pathological variability during standing and walking. Neurosci Biobehav Rev, 68:111-119.

Konig N, Singh NB, Baumann CR, Taylor WR (2016). Can Gait Signatures Provide Quantitative Measures for Aiding Clinical Decision-Making? A Systematic Meta-Analysis of Gait Variability Behavior in Patients 
with Parkinson's Disease. Front Hum Neurosci, 10:319.

[43] Chen KL, Xu Y, Chu AQ, Ding D, Liang XN, Nasreddine ZS, et al. (2016). Validation of the Chinese version of Montreal Cognitive Assessment basic for screening mild cognitive impairment. Journal of the American Geriatrics Society, 64.

[44] Yu X, Tam WW, Wong PT, Lam TH, Stewart SM (2012). The Patient Health Questionnaire-9 for measuring depressive symptoms among the general population in Hong Kong. Comprehensive psychiatry, 53:95-102.

[45] Mary Elizabeth H, Linda JW, Louise CH, John TC (2004). A Short Scale for Measuring Loneliness in Large Surveys: Results From Two Population-Based Studies. Research on Aging, 26:655-672.

[46] Schwarzer R, Jerusalem M. 1995. Generalized SelfEfficacy Scale. J. Weinman, S Wright, and M Johnson (eds.) Measures in health psychology: A user's portfolio, Causal and control beliefs, editor. Windsor England: NFER-NELSON. 35-37.

[47] Smith BW, Dalen J, Wiggins K, Tooley E, Christopher P, Bernard J (2008). The brief resilience scale: assessing the ability to bounce back. Int J Behav Med, 15:194-200.

[48] Rabin R, Charro Fd (2001). EQ-SD: a measure of health status from the EuroQol Group. Annals of medicine, 33:337-343.

[49] Lamoureux EL, Pallant JF, Pesudovs K, Hassell JB, Keeffe JE (2006). The Impact of Vision Impairment Questionnaire: an evaluation of its measurement properties using Rasch analysis. Investigative ophthalmology \& visual science, 47:4732-4741.

[50] Jupiter T, Palagonia CL (2001). The Hearing Handicap Inventory for the Elderly screening version adapted for use with elderly Chinese American individuals. American Journal of Audiology, 10:99-103.

[51] Collin C, Wade DT, Davies S, Horne V (1988). The Barthel ADL Index: A reliability study. International Disability Studies, 10:61-63.

[52] Lawton MP, Brody EM (1969). Assessment of older people: self-maintaining and instrumental activities of daily living. Gerontologist, 9:179-186.

[53] Ng TP, Feng L, Nyunt MSZ, Larbi A, Yap KB (2014). Frailty in older persons: multisystem risk factors and the Frailty Risk Index (FRI). Journal of the American Medical Directors Association, 15:635-642.

[54] Neelakantan N, Whitton C, Seah S, Koh H, Rebello SA, Lim JY, et al. (2016). Development of a SemiQuantitative Food Frequency Questionnaire to Assess the Dietary Intake of a Multi-Ethnic Urban Asian Population. Nutrients, 8.

[55] Strawbridge WJ, Wallhagen MI, Shema SJ (2007). Impact of spouse vision impairment on partner health and well-being: A longitudinal analysis of couples. The Journals of Gerontology Series B: Psychological Sciences and Social Sciences, 62:S315-S322.

[56] Lempers JD, Clark-Lempers D, Simons RL (1989). Economic hardship, parenting, and distress in adolescence. Child development:25-39.
[57]

[59] Ng A, Chew I, Narasimhalu K, Kandiah N (2013). Effectiveness of Montreal Cognitive Assessment for the diagnosis of mild cognitive impairment and mild Alzheimer's disease in Singapore. Singapore Med J, 54:616-619.

[60] Sung SC, Low CC, Fung DS, Chan YH (2013). Screening for major and minor depression in a multiethnic sample of Asian primary care patients: a comparison of the nine-item Patient Health Questionnaire (PHQ-9) and the 16-item Quick Inventory of Depressive Symptomatology - SelfReport (QIDS-SR16). Asia Pac Psychiatry, 5:249-258. [61] Chan KM, Pang WS, Ee CH, Ding YY, Choo P (1999). Functional status of the elderly in Singapore. Singapore Med J, 40:635-638.

[62] Ng TP, Niti M, Chiam PC, Kua EH (2006). Physical and cognitive domains of the Instrumental Activities of Daily Living: validation in a multiethnic population of Asian older adults. J Gerontol A Biol Sci Med Sci, 61:726-735.

[63] Ge L, Yap CW, Ong R, Heng BH (2017). Social isolation, loneliness and their relationships with depressive symptoms: A population-based study. PLoS One, 12:e0182145.

[64] Fenwick EK, Man RE, Rees G, Keeffe J, Wong TY, Lamoureux EL (2017). Reducing respondent burden: validation of the Brief Impact of Vision Impairment questionnaire. Qual Life Res, 26:479-488.

[65] Luo N, Chew LH, Fong KY, Koh DR, Ng SC, Yoon $\mathrm{KH}$, et al. (2003). Validity and reliability of the EQ5D self-report questionnaire in English-speaking Asian patients with rheumatic diseases in Singapore. Qual Life Res, 12:87-92.

[66] Wee HL, Loke WC, Li SC, Fong KY, Cheung YB, Machin D, et al. (2007). Cross-cultural adaptation and validation of Singapore Malay and Tamil versions of the EQ-5D. Ann Acad Med Singapore, 36:403-408.

[67] Tan ML, Wee HL, Salim A, Lee J, Ma S, Heng D, et al. (2016). Validity of a Revised Short Form-12 Health Survey Version 2 in Different Ethnic Populations. Ann Acad Med Singapore, 45:228-236.

[68] Tham YC, Lim SH, Shi Y, Chee ML, Zheng YF, Chua $\mathrm{J}$, et al. (2018). Trends of Visual Impairment and Blindness in the Singapore Chinese Population over a Decade. Sci Rep, 8:12224.

[69] Zheng Y, Lavanya R, Wu R, Wong WL, Wang JJ, Mitchell P, et al. (2011). Prevalence and causes of visual impairment and blindness in an urban Indian population: the Singapore Indian Eye Study. Ophthalmology, 118:1798-1804.

[70] Rubin GS, West SK, Munoz B, Bandeen-Roche K, Zeger S, Schein O, et al. (1997). A comprehensive 
assessment of visual impairment in a population of older Americans. The SEE Study. Salisbury Eye Evaluation Project. Invest Ophthalmol Vis Sci, 38:557-568

[71] Swenor BK, Simonsick EM, Ferrucci L, Newman AB, Rubin S, Wilson V (2015). Visual impairment and incident mobility limitations: the health, aging and body composition study. J Am Geriatr Soc, 63:46-54.

[72] West SK, Rubin GS, Broman AT, Munoz B, BandeenRoche K, Turano K (2002). How does visual impairment affect performance on tasks of everyday life? The SEE Project. Salisbury Eye Evaluation. Arch Ophthalmol, 120:774-780.

[73] Samper-Ternent R, Al Snih S (2012). Obesity in Older Adults: Epidemiology and Implications for Disability and Disease. Rev Clin Gerontol, 22:10-34.

[74] Lim KH, Jasvindar K, Cheong SM, Ho BK, Lim HL, Teh CH, et al. (2016). Prevalence of smoking and its associated factors with smoking among elderly smokers in Malaysia: findings from a nationwide population-based study. Tob Induc Dis, 14:8.

[75] (2000). Obesity: preventing and managing the global epidemic. Report of a WHO consultation. World Health Organ Tech Rep Ser, 894:i-xii, 1-253.

[76] Misra A, Vikram NK, Gupta R, Pandey RM, Wasir JS, Gupta VP (2006). Waist circumference cutoff points and action levels for Asian Indians for identification of abdominal obesity. Int J Obes (Lond), 30:106-111.

[77] Manolio TA, Fishel SC, Beattie C, Torres J, Christopherson R, Merritt WT, et al. (1988). Evaluation of the Dinamap continuous blood pressure monitor. Am J Hypertens, 1:161s-167s.

[78] Sun C, Liew G, Wang JJ, Mitchell P, Saw SM, Aung $\mathrm{T}$, et al. (2008). Retinal vascular caliber, blood pressure, and cardiovascular risk factors in an Asian population: the Singapore Malay Eye Study. Invest Ophthalmol Vis Sci, 49:1784-1790.

[79] Wong TY, Cheung N, Tay WT, Wang JJ, Aung T, Saw $\mathrm{SM}$, et al. (2008). Prevalence and risk factors for diabetic retinopathy: the Singapore Malay Eye Study. Ophthalmology, 115:1869-1875.

[80] (2002). K/DOQI clinical practice guidelines for chronic kidney disease: evaluation, classification, and stratification. Am J Kidney Dis, 39:S1-266.

[81] Levey AS, Stevens LA, Schmid CH, Zhang YL, Castro $\mathrm{AF}, 3 \mathrm{rd}$, Feldman HI, et al. (2009). A new equation to estimate glomerular filtration rate. Ann Intern Med, 150:604-612.

[82] Bowie CR, Harvey PD (2006). Administration and interpretation of the Trail Making Test. Nat Protoc, 1:2277-2281.

[83] Foster PJ, Oen FT, Machin D, Ng TP, Devereux JG, Johnson GJ, et al. (2000). The prevalence of glaucoma in Chinese residents of Singapore: a cross-sectional population survey of the Tanjong Pagar district. Arch Ophthalmol, 118:1105-1111.

[84] Baskaran M, Foo RC, Cheng CY, Narayanaswamy AK, Zheng YF, Wu R, et al. (2015). The Prevalence and Types of Glaucoma in an Urban Chinese Population: The Singapore Chinese Eye Study. JAMA Ophthalmol,
133:874-880.

[85] Foster PJ, Buhrmann R, Quigley HA, Johnson GJ (2002). The definition and classification of glaucoma in prevalence surveys. Br J Ophthalmol, 86:238-242.

[86] Spaeth GL (1971). The normal development of the human anterior chamber angle: a new system of descriptive grading. Trans Ophthalmol Soc U K, 91:709-739.

[87] Scheie HG (1957). Width and pigmentation of the angle of the anterior chamber; a system of grading by gonioscopy. AMA Arch Ophthalmol, 58:510-512.

[88] (1991). Grading diabetic retinopathy from stereoscopic color fundus photographs--an extension of the modified Airlie House classification. ETDRS report number 10. Early Treatment Diabetic Retinopathy Study Research Group. Ophthalmology, 98:786-806.

[89] Klein R, Davis MD, Magli YL, Segal P, Klein BE, Hubbard L (1991). The Wisconsin age-related maculopathy grading system. Ophthalmology, 98:1128-1134.

[90] Chylack LT, Jr., Wolfe JK, Singer DM, Leske MC, Bullimore MA, Bailey IL, et al. (1993). The Lens Opacities Classification System III. The Longitudinal Study of Cataract Study Group. Arch Ophthalmol, 111:831-836.

[91] (1994). Assessment of fracture risk and its application to screening for postmenopausal osteoporosis. Report of a WHO Study Group. World Health Organ Tech Rep Ser, 843:1-129.

[92] Ong HL, Abdin E, Chua BY, Zhang Y, Seow E, Vaingankar JA, et al. (2017). Hand-grip strength among older adults in Singapore: a comparison with international norms and associative factors. BMC Geriatr, 17:176.

[93] Mathers C, Smith A, Concha M (2000). Global burden of hearing loss in the year 2000. Global burden of Disease, 18:1-30.

[94] Rumeau C, Nguyen DT, Jankowski R (2016). How to assess olfactory performance with the Sniffin' Sticks test((R)). Eur Ann Otorhinolaryngol Head Neck Dis, 133:203-206.

[95] Schimmel M, Christou P, Miyazaki H, Halazonetis D, Herrmann FR, Muller F (2015). A novel colourimetric technique to assess chewing function using twocoloured specimens: Validation and application. J Dent, 43:955-964.

[96] Lang NP, Adler R, Joss A, Nyman S (1990). Absence of bleeding on probing. An indicator of periodontal stability. J Clin Periodontol, 17:714-721.

[97] Wang X, Lamoureux E, Zheng Y, Ang M, Wong TY, Luo N (2014). Health burden associated with visual impairment in Singapore: the Singapore epidemiology of eye disease study. Ophthalmology, 121:1837-1842.

[98] Stalvey BT, Owsley C, Sloane ME, Ball K (1999). The Life Space Questionnaire: A measure of the extent of mobility of older adults. Journal of Applied Gerontology, 18:460-478.

[99] Chiu CT, Malhotra R, Tan SM, Lim J, Chan A, Teoh $\mathrm{KH}$, et al. (2017). Dental health status of community- 
dwelling older Singaporeans: findings from a nationally representative survey. Gerodontology, 34:57-67.

[100] Eisbruch A, Kim HM, Terrell JE, Marsh LH, Dawson
LA, Ship JA (2001). Xerostomia and its predictors following parotid-sparing irradiation of head-andneck cancer. Int J Radiat Oncol Biol Phys, 50:695-704. 